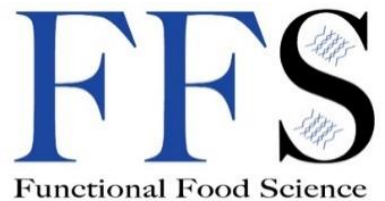

\title{
Classification and regulation of functional food proposed by the Functional Food Center
}

\author{
Danik Martirosyan*1,2, Trevor Lampert ${ }^{1,3}$, Morgan Ekblad ${ }^{1,4}$
}

${ }^{1}$ Functional Food Center, Dallas, TX, USA; ${ }^{2}$ Functional Food Institute, San Diego, CA, USA; ${ }^{3}$ National University of Natural Medicine, Portland, Oregon, USA; ${ }^{4}$ University of South Florida Tampa, Florida, USA

*Corresponding Author: Danik Martirosyan, PhD, Functional Food Institute, San Diego, CA, USA

Submission Date: January 10 ${ }^{\text {th }}, 2022$; Acceptance Date: February $3^{\text {rd }}, 2022$; Publication Date: February $7^{\text {th }}, 2022$

Please cite this article as: Martirosyan D.M., Lampert T., Ekblad M. Classification and regulation of functional food proposed by the functional food center. Functional Food Science 2022; 2(2): 25-46. DOI: https://www.doi.org/10.31989/ ffs.v2i2.890

\section{ABSTRACT}

There has yet to be a consensus on the definition of functional foods (FF), and accordingly many institutions lack a comprehensive process for its classification. The Functional Food Center (FFC) has previously proposed a multi-step process for the development of functional food products and ways by which to bring them to market without means to classify established items. This article is advancing the previously proposed methodology with the addition of new steps. The newest steps focus on themes of transparency by the publishing of peer-reviewed articles for the functional food product as mandatory for accreditation. In doing so, this will provide greater access to information for the functional food market, as well as, acceptance and trustworthiness of functional claims. Additionally, the Functional Food Center has created a new system for categorizing functional foods. The new categorization system uses improved research on epidemiological and after market studies, and evaluates the quality of evidence for the functional food product (FFP) as A, B, or C. A classification of A denotes the completion of aftermarket research, epidemiological studies, and certification 
of functional food status. Classification B denotes completion of epidemiological studies and certification of functional food status. Lastly, C indicates that the product has only been certified as functional. The Functional Food Center's definition of functional foods, steps on how to create functional foods, and proposed categorization will help to describe our proposed regulation of FFP.

KEYWORDS: Functional Food Classification, Functional Food Regulation, Functional Food Products, Categorization Grades of Functional Food, How to Create Functional Food, Bioactive Compounds, Functional Food Definition

\section{Revised Regulation with Integrated Classification Labels}

Previously Established

Steps 1-9. (i.e. establish bioactive compounds, health benefits,

biomarkers, dosages, and food vehicles)

\section{New Step 10; Data published in open access to the public (i.e. peer-reviewed journals)}

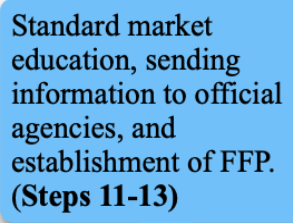

Standard market education, sending information to official agencies, and establishment of FFP.

(Steps 11-13)

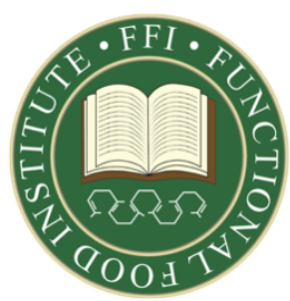

FFP is released to market in Step 14 with new classification of 'C'.

- Increase communication concerning FFP's between the FFC, official agencies, researchers, and the public.

- Provide the public with easy to read labels to denote where in the process a FFP is.

- Emphasize larger studies after establishment to classify FFP's further for public use.

- Build of off previous FDA claims to foster relations with the FFC and legitimize FFP's further.
FFP is subject to epidemiological studies in Step 15. New classification of ' $B$ ' is provided upon completion.

FFP is subject to aftermarket studies in Step 16. New classification of ' $A$ ' is provided upon completion.
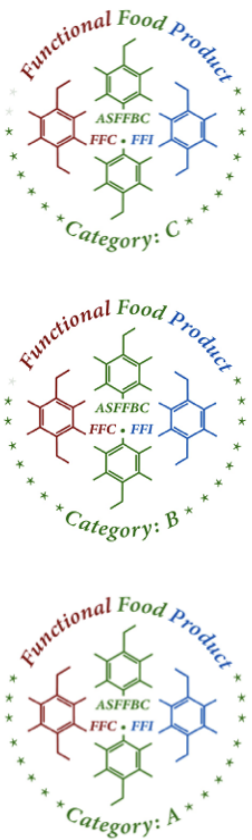

CFFC 2022. This is an Open Access article distributed under the terms of the Creative Commons Attribution 4.0 License (http://creativecommons.org/licenses/by/4.0) 


\section{INTRODUCTION}

Chronic disease in the United States (U.S.) is highly prevalent and is projected to increase over the next several decades [1]. In the United States, chronic diseases are among the most costly health conditions [1]. The ever-growing chronically ill population together with skyrocketing medical costs are an urgent problem, providing an opportunity to manage disease and disease progression using non-medical means [2]. Academic institutions, government agencies, and researchers around the globe are dedicating a great amount of effort to identifying how FFs and food ingredients might help prevent disease progression, or optimize health, thereby reducing healthcare costs and improving the quality of life for many [3].

In order to supply consumers with safe and effective FFPs, it is necessary for government agencies to formally agree on a comprehensive definition. The FFC proposes a definition for FFs as "Natural or processed foods that contain biologically-active compounds, which, in defined, effective, non-toxic amounts, provide a clinically proven and documented health benefit utilizing specific biomarkers, to promote optimal health and reduce the risk of chronic/viral diseases and manage their symptoms" [3]. Furthermore, FFs must undergo an evaluation, including the publishing of peer reviewed journals, to ensure only safe and effective products will be released to the market [4].

The Japanese Foods for Specific Health Uses (FOSHU) started in 1991 and has the potential to serve as a guide to the developing policies for FFs. FOSHU is the most comprehensive FF system in Japan [5]. The FOSHU system evaluates foods with a claim that identifies the product is suited for specified health uses and is evaluated and approved by the Consumer Affairs Agency (CAA) [5]. The claim of "specific health uses" is in reference to the promotion or maintenance of health; including blood pressure, or blood cholesterol [6]. Each FFP is approved for FOSHU status after going through a review of safety and efficacy. The FOSHU approval system requires the identification of at least one active ingredient to which the claims may be attributed [6]. FOSHU approved products receive a logo that clearly states "Approved by Consumer Affairs Agency" and "Food for Specified Health Uses" [2]. Many institutions across the globe are incorporating fundamental principles of FOSHU into their own regulatory framework.

In the United States, the Food and Drug Administration (FDA) evaluates and authorizes health claim petitions for food [7]. Unfortunately, there is yet to be a formal definition of FFs or even a distinct FF category [8]. The FFC and the Academic Society of Functional Foods and Bioactive Compounds (ASFFBC) are in communication with the FDA and other governmental agencies to define, classify and regulate FFs [2]. Furthermore, the FFC has communicated with government officials in the United States congress for advice relating specifically to the implementation of foods within the field of health. As an example, the FFC and the United States Department of Agriculture (USDA) organized a joint international conference at the University of San Diego in 2014 to explore FFs and their bioactive compounds. Furthermore, one year later in 2015, the pair organized an international conference with Kobe University in Japan involving both USDA and FOSHU representatives. In accordance with an emphasis on global precedence, the FFC organized four international conferences in conjunction with the Beth Israel Deaconess Medical Center (BIDMC) at Harvard Medical School, Boston, MA from 2015 to 2018. At these conferences, FFs and chronic diseases were explored with special lecturers from the FDA, National Institutes of Health (NIH), and USDA. Each gathering saw that the FFC 
recognized the current state of FF's definition, creation, regulation, and safety in order to expand the field and not stagnate.

This paper proposes a definition, classification, and regulation of FFs as well as a new approach of how to create FFPs and bring them to market. Functional food development is a long process but ideally one that can serve as a guide for scientists and researchers all around the world to develop their FFPs to better serve practitioners and consumers alike. The classification and regulation of FF will positively contribute to human health and well-being in the United States and across the globe.

Regulation of Functional Food Products: Latterly, FFs have had a multitude of definitions. Older definitions have been updated as the knowledge surrounding bioactive compounds and function in managing health outcomes has been expanded. Furthermore, variability in the interpretation of some definitions may be related to national differences. The FFC seeks to better establish a system for classifying and regulating FFs for the public of the United States. To do this, the FFC has defined FFs as "natural or processed foods that contain biologicallyactive compounds; which, in defined, effective, non-toxic amounts, provide a clinically proven and documented health benefit utilizing specific biomarkers, to promote optimal health and reduce the risk of chronic/viral diseases and manage their symptoms" [3]. This definition will serve as a basis for classification. If a functional food does not meet these parameters, then it cannot be classified as functional food. To discern the viability of FFs that promote health and well-being, bioactive compounds are analyzed to better justify a food's use. Their capacity for promoting optimal health is what confers their value. Promotion occurs through specific compounds or nutrients within the food vehicle being utilized by the body in a specific way. Bioactive compounds affect the body by managing cell metabolism and functions, promoting health in certain conditions. The efficacy of such processes is managed by observing the prevalence of biomarkers within the body. By manipulating biomarkers that have a sustained action on biological processes within the body, bioactive compounds can better demonstrate promotion of health through precise interactions [9]. The discernment of bioactive compound efficacy within given foods is the primary foundation by which FFs can be analyzed. Functionality is referenced almost exclusively by the action of these bioactive compounds on biomarkers, meaning that without them, food does not meet the requirements for functionality. Should the food not be certifiably functional due to its bioactive compound content, the most one can hope to gain from it is the associated nutrient value. Without bioactive compounds as the foundation for FF analysis, the scientific field would have little empirical basis to analyze health outcomes due to a lack of causational review. The FFC has suggested a 16-step course for the induction of foods into the category of functional food. This is an update to the current literature published, with the previous 15 step plan being adjusted for greater refinement of the process to promote sustainable and trustworthy products [3]. The key step differentiating the old system from the updated one is the inclusion of publications for relevant bioactive compounds or FFs containing those substances being promoted in open access to peer-reviewed journals, the public, and governmental agencies. By doing this, transparency is promoted in hopes that the compound can continue to be scrutinized against other forms of literature to best determine if it is functional. The FFC outlines the system to achieve a FFP as follows (Table 1): 
Table 1. Steps to develop functional food products and bring them to the market

\begin{tabular}{|c|c|}
\hline $\begin{array}{l}\text { Step } \\
\text { Number }\end{array}$ & Description of Steps to create FF Products \\
\hline 1 & Establishes a goal of the functional food product, \\
\hline 2 & Determines relevant bioactive compound(s), \\
\hline 3 & Establishes the appropriate dosage of bioactive compound(s), \\
\hline 4 & Determines the specific pathway and mechanism of action, \\
\hline 5 & Establishes relevant biomarker(s), \\
\hline 6 & Chooses an appropriate food vehicle for bioactive compound(s), \\
\hline 7 & Provides preclinical studies one efficacy and safety, \\
\hline 8 & Provides clinical trials for dosage, efficacy, and safety, \\
\hline 9 & Creates a special label that informs the consumers of the most effective way to consume the product, \\
\hline 10 & Publications are submitted to peer-reviewed journals, preferably in open access, \\
\hline 11 & Educates the general public, \\
\hline 12 & Sends information to credible governmental agencies, such as the FDA, for approval, \\
\hline 13 & Official establishment of the accredited functional food product, \\
\hline 14 & Release the functional food product to the market. (Receive the basic category (level C)), \\
\hline 15 & Provides epidemiological studies. (Reapply for the approval for a new category (level B)), \\
\hline 16 & Provides after market research. (Reapply for the approval for a new category (level A)). \\
\hline
\end{tabular}

Step 1: The first step in the updated 16 stage plan as displayed in Table 1 is the establishment of goals for a particular FFP. This, as seen in previous iterations of the plan, remains relatively the same. Purposes may include helping to reduce the risk of developing chronic diseases or viral afflictions, managing the symptoms of specific conditions, enhancing bodily performance, or simply promoting optimal health. To decide on such, past studies in different academic fields are called upon to influence and determine the feasibility of such a product.
Suggestions may come from fields not associated with nutrition, as studies in ethnobotany and anthropology may suggest promising data that has not been explored yet [3]. In this stage, specificity is paramount in proceeding further, as a clear and concise methodology needs to be explored in order to pursue research and certification in the most efficient way. Without this specificity and precise preliminary research, the FF in question may not have suggestive data that indicates a possible future use. Alternatively, the use may be 
misconstrued and be applicable to a different affliction entirely. Previous iterations of the process have displayed this step as phytonutrient analysis [10]. When simplified, the following steps prior to pre-clinical testing are still relatively new when compared to former iterations.

Step 2: The second step revolves around determining the relevant bioactive compounds within the FFP. After a preliminary assessment of the FFP, its bioactive compounds must be inspected and researched. Identifying physiologically active bioactive compounds is an important element of classifying FFs. Bioactive compounds are present in greater amounts in FFPs and their effect on human health is being continuously investigated when compared to normal food items [11]. Bioactive compounds-such as resveratrol, epigallocatechin, curcumin, oleuropein, sulforaphane, quercetin, ellagic acid, anthocyanins, beta-glucans, and other biomolecules-have been studied as factors associated with the beneficial pathophysiology for cardiovascular diseases, diabetes, metabolic syndrome, and cancer. [12-14]. The study on the protective and health-promoting effects of bioactive compounds has made these components particularly important for the categorization of FFs [15]. The isolation of bioactive compounds from plant or animal sources still remains difficult since extracts often include a mixture of several kinds of bioactive compounds or phytochemicals [16]. Many analytical, chromatographic, and molecular approaches are used to fingerprint bioactive chemicals, with an emphasis on high-performance liquid chromatography/high performance thin layer chromatography, Fourier-transform infrared spectroscopy, and immunoassay techniques [17]. Once identified, aspects such as dosage and mechanisms of action can be ascertained [3]. Relevant bioactive compounds are important to identify as these could possibly be better suited as additives in processed FFPs, or be found in different food vehicles. These are all aspects that need to be considered when rationalizing the use of bioactive compounds.

Step 3: As mentioned in Table 1, the third step involves establishing an appropriate dosage for the identified bioactive compound [3]. Dosage is important as it is defined by the therapeutic range in which a compound may exhibit positive effects in users. Biological activity has been acknowledged with bioactive compounds that have a positive effect, but negative effects are also a form of bioactivity [18]. This includes adverse effects such as toxicity, allergenicity, and mutagenicity, which are usually dependent on the dose and bioavailability of a given substance. An important question when defining FFs with respect to the bioactive compounds is whether or not they propose a risk from a safety perspective. In fact, evidence exists regarding the risk of consuming an excessive amount of healthy substances such as antioxidants, omega 3, and soy isoflavones [19]. Should dosages exceed the toxic threshold or not reach the therapeutic range, then the bioactive compound will have adverse or minimal to no effects respectively. Each bioactive compound's functionality is different, meaning the way by which they affect the body is different as well. Parameters set in place for one may not be applicable for the other, meaning dosage may be altered at the discretion of the research team to promote observable effects. The process by which the dosage is established is at the discretion of the research team. Furthermore, due to intrinsic differences, it is important to fully understand the bioactive compound's functionality completely before moving onto the later stages, determining the specific pathway and mechanism of action.

Step 4: In the fourth step, the plausible mechanism of action is explored, as well as its intended pathway [3]. 
Bioactive compounds perform their activities through interaction with specific biologic mechanisms and the synergistic effect of other nutrients and food matrix components could be involved in the described effect [20]. Mechanisms of action of bioactive compounds may be multiple, especially in the food matrix [20]. This issue becomes complex because food ingredients and bioactive compounds may interact, enhancing their desirable or undesirable effects, which is not the case for a single bioactive compound administered as an extract [20]. Accounting for this complexity, the mechanism(s) of action of the bioactive compounds needs to be adequately characterized, as a prerequisite for a given claim for its authorization. In this line, in order to accept a specific health claim, the science supporting it should contain all information on a plausible mechanism(s) of action of the given bioactive compounds. Without such documentation, the application of a bioactive compound in risk reduction, managing symptoms, or promoting optimal health cannot be empirically justified. Discerning the mechanisms behind how a bioactive compound acts allow for methodologies to be understood, possibly affecting how future clinical/epidemiological trials are conducted. An important aspect at this stage is characterized by how the compound in question interacts with other constituents of food in the food matrix. Should any negative interactions between the compound and others be observed, the FF's development may be terminated, as it could be potentially dangerous without proper, strict, regulation.

Step 5: Once these factors have been established, then the relevant biomarker can be confirmed in the fifth step. Biomarkers are indicators within the body that should, in theory, be adjusted through the application of the bioactive compound. Essentially, they act as confirmation that the bioactive compound is working as intended [3]. Nutritional studies must use a variety of biomarkers that measure overall health conditions [21]. Often, the biomarkers are linked specifically to the affliction, allowing progress to be observed as compounds are administered. To assess subcellular, cellular, tissue, and whole-body interactions with bioactive compounds, an integrated approach incorporating ideas and data from nutritional sciences, food science, molecular biology, biochemistry, and plant science is required [22]. Once the mechanisms and pathways are established, biomarkers may become more evident through their relationship with the pathogenesis of conditions. Biomarkers, are indicative of certain conditions and specific to affiliated diseases. As observed in managing hypertension, four unique biomarkers were observed and manipulated to induce change [9]. Without the observance of such markers, the viability of bioactive compounds cannot be justified as their implications cannot be quantified. In establishing relevant biomarkers, scientists can confirm the causal effect by which the FF activates. Without such confirmation, the ensuing clinical and epidemiological studies have no means to confirm whether the FFP supports its intended use or not.

Step 6: In the sixth step, a suitable food vehicle needs to be decided upon. There are different means by which ingestion may occur. Several food items may contain desirably potent amounts of bioactive compounds in question; however, availability and other intrinsic factors need to be accounted for and rationalized against [3]. There is also the possibility that the bioactive compound in question is best suited for use as an additive to a food item that does not typically contain desired amounts of the bioactive compound. The prospect of adding the bioactive compound to a food item, and therefore creating a processed FF, could serve to benefit the user more, depending on the application. This would require the newly postulated item to resubmit itself to the system, starting over. Naturally, the processed FF item 
cannot be certifiably functional until it completes the 16 step process, however, the indication of benefits can provide just cause for it to be subject to the initial six steps again. Furthermore, there are many cultural/social implications to consider when discerning which food vehicle is best suited. Due to ethnic or religious reasons, some food vehicles may be ill-equipped for consumption in specific populations. Other intrinsic properties may be cited, such as allergenic effects. Prior research into the topic has yielded seven basic criteria that need to be considered: a) The food must be consumed by a large enough portion of the population to confer benefit; b) the food must contain the appropriate amount of bioactive compound to detect the benefit, taking into consideration intake from other common dietary sources; c) the food must target a specific condition; d) the active compound(s) must remain stable through packaging, distribution, storage, and consumption processes; e) the nutrients should be easily available from the food vehicle; f) fortification of the compound into the food should not make it undesirable to consume; g) and the cost should be reasonable for consumers [23]. All of these factors must be simultaneously considered so that the consumer ingests the most desirable product in optimal amounts. This step's placement is drastically different from early iterations, signifying the continuous evolution of this process. In earlier procedures, the development of food vehicles was reserved for implementation after clinical trials [10].

\section{Pre-clinical and clinical testing}

Step 7: The seventh step allows preclinical studies to determine efficacy and safety to a greater extent once baseline technicalities have been met and approved. These and the following clinical trials represent the halfway point in the prospective FF approval process as seen in Table 1. Without passing this checkpoint, the FF cannot progress on the course for approval without refining or adjusting previous steps [3]. To determine efficacy and safety, in vitro and vivo studies utilize animals to cross-reference dosages and safety to ascertain effective dosages without adverse side effects. This is done prior to human trials in order to take every precaution possible. The application of food items in this way should have no effect on the individual; however, with doses exceeding normal, preventing adverse issues will only serve to benefit future consumers. This step remains relatively consistent with previous iterations, as it is an important prerequisite to determine functional use and safety later in clinical trials [10].

Step 8: The eighth step provides human clinical trials, as efficacy and safety have now been preliminarily assured. The dosage for the administration of the bioactive compound is refined further, narrowing the specificity of what can be administered safely in humans for the greatest therapeutic benefit. In this stage, adverse effects are noted and analyzed to determine the viability of pursuing future use of the compound [3]. Should adverse effects be too common or debilitating, then the pursuit of the application of the compound may be halted. This is reminiscent of previous definitions for this step. As with earlier renditions, human-appropriate dosages are tested for adverse side-effects and require pre-clinical trials as prerequisites [10]. Additionally, clinical trials may demonstrate that the compound is not as effective as stipulated previously. If no clear benefit is observed by analyzing the relevant biomarkers, then the progress of development may be halted until more compelling evidence is observed. Clinical trials as overseen in the outlined process would need to be standardized with set regulations. The location in which they take place is of no concern, as long as standards set in place by the FFC at a later date are met. This is meant so that each bioactive compound and food vehicle can be judged based on similar criteria, excluding bias to the 
fullest extent. An absence of regulations may allow for faulty products to reach the market at an endorsed level, creating liability both on part of the FFC and FDA.

\section{Labeling of functional food product}

Step 9: In the ninth step, a label corresponding to the observed therapeutic dosages may be created only once human clinical trials prove effective. This label will define intake levels and instructions for ingestion, correlating most commonly with ingestion methods used in clinical studies. This label is mostly for the consumers' benefit, as it will outline the necessities for ingestion. Labeling includes date of approval, observable health benefits, daily recommended usage, identification of the bioactive substance, consumption method, precautions regarding consumption, and amount of bioactive substance [3]. Each of these is to ensure that the consumer understands the processes and implications of ingesting the bioactive compound and food vehicle. As an example, the FOSHU system in Japan already has an established label for FFPs. On the already established FOSHU labels, the product specification is guaranteed at the time of consumption, meaning that the label contains an accurate measure for how much of a specific bioactive compound is contained within the food vehicle. Furthermore, the label can be specific about the food product's function. There are five categories by which a FF can be labeled under FOSHU, including: regular, standardized, risk-reduction, reauthorized, and qualified [2]. Regular FOSHU goes through a full evaluation process as they have an applicable ingredient. Standardized FOSHU only contains ingredients that adhere to governmental standards concerning the dosage. Risk-reduction FOSHU refers to compounds that have been proven to reduce the risk of disease. Reauthorized FOSHU are products that have already been confirmed and evaluated but want to change a characteristic such as a flavor. Qualified FOSHU are products that contain an ingredient with an unknown mechanism observed to exhibit health benefits. Each of these classifies the product as having gone through portions of the certification process under FOSHU or specifically pertaining to certifiable attributes possessed by the FF. The FFC label will include similar information; however, it will utilize identifiers from the newly proposed ' $A B C$ ' classification system, outlined later in steps 14-16. Creation of an appropriate label for FFPs is a newer concept instituted in the process. Previous outlines have not placed emphasis on a step solely meant for developing a label to be used by the public and manufacturers prior to education and marketing [10]. Though not specifically outlined, the concept has been cited as a major constituent for the process. As cited previously, a special food label is required for the differentiation of FFPs amongst other items that can be accepted by the American public [24]. Although this pertains more heavily to the acceptance of $F F$, it is still applicable in how a product would need to be approved.

The actual placement and design of the label are also important, prompting an easy-to-read design as displayed later in the article. Labels on the front of food products that are specific to that food, instead of diet, are observed to be the most effective at communicating benefits. Adapted from label diet-directive presentations on both healthy and unhealthy food, FFC labels discerning the score of FF products on foods that promote health evoke more positive behavioral responses [25]. Diet-directives may be advertised on both healthy and unhealthy food items. The FFC label, as indicated in Figure's 1, 2, and 3, differ from these as they advertise specific benefits, not falling to heuristics of critical thinking requirements, memory recall, and decision making [25]. Thus, consumers are informed better, prompting them to actively consider the implications of FF effects. Inspiration taken from the simplicity of the FOSHU label would allow for the FFC's FFPs to be easily identified and analyzed by consumers. 
Open access for the public

Step 10: This tenth step of the system is newly postulated and is meant to give greater access to information for expansion, disputation, or acceptance. Open access to information by publishing in peer-reviewed journals will make information more accessible to the consumer and seem legitimate to governmental agencies. While this step cannot be mandatory for furtherance in the process, it is highly recommended. Open access is most desired as it promotes transparency and allows anyone interested to view findings and offer an opinion. There may be some outstanding variables that do not allow for open access, so publication in closed access journals will suffice. Regardless, some form of peer-reviewed publication needs to be met to continue the FFPs certification. Outlets by which information is published should revolve around some aspect of nutrition, food science, or functional food science. In peer-reviewing articles or studies committed, the literature surrounding specific bioactive compounds can be expanded, helping to legitimize not only the use of specific bioactive compounds analyzed but prompting the studying of others. In allowing research to be made accessible to the public, the next stage of educating the market can have a reasonable platform to start from. It has been observed that for acceptance to become resolute, supportive data is often needed to legitimize information. This is consistent with FF items within the proposed classification system. Especially with food, items within a system need to facilitate consumer trust to be successful. It has been established that product assurance, such as certification, labels, and health claims, facilitates growth of consumer trust [26]. Each of these aspects is an integral part of the process proposed as an update to the current literature. Thus, consumer trust may be broadened through open access literature and direct means of data access on part of the public. Should open access not be an option for some articles, then trust in agencies and others in the academic community will need to suffice. These participants may be those previously alluded to in the FFC's academic society framework. By allowing not only other agencies to have access to the information, but peer-reviewed journals as well, the public can more readily trust the process.

Step 11: In the eleventh step, specifics of the FF and its bioactive compounds will be communicated to the public. Indicators and biomarkers will be explained regarding the ways in which they interact with the FF and associated bioactive compound [3]. Information will also be specific for the consumer, conveying pertinent aspects of the FF in a relatable way. Any relevant information that the consumer would need to understand in order to ingest the product safely, should be communicated. Furthermore, this step will allow people to understand what can be expected from this FF. By being introduced to the product and becoming educated about its properties, the public can make better informed decisions to complement and improve their consumption habits. Ultimately, it is hoped that these guidelines can help create an intelligent and informed market that fully understands the implications of consuming bioactive compounds.

Step 12: Under the twelfth step, the data gathered up until this point is sent to third parties and governmental agencies for refinement and approval [3]. Submissions should primarily be directed by the FFC to the FDA, as these organizations are the handlers of FFs and health claims respectively. As outlined in Figure 4, the FDA must be submitted regardless of prior health claims. While recommended, they are not necessary. This is due to FFP producers now wishing for their product to be certifiably functional. The FDA will analyze any claims with evidence so that the FFPs in question may be approved. To clarify, the FFC will act as a mediator between the applicant and 
FDA, providing apt recommendations based on prior analyses in the updated 16-step process. This step is crucial in building trust with the public as it essentially validates the food product and its benefits by other official agencies. Public mistrust in already established food verification systems requires more transparent and scientifically backed procedures to be instituted. Transparent scientific communication with an official body can engender consumer confidence for the product in question [27]. This cooperation will lead to the acceptance of the new product by both official agencies and the public. Some of the earliest iterations of this process did not include the involvement of governmental agencies whatsoever [3]. They acted more like an afterthought, aligning more with the required processes for validating the field instead of a singular product [10]. This may have resulted in some hesitation by the public up until this proposal.

Step 13: The thirteenth step sees the official establishment of the accredited FFP by the FFC and FDA. With this step, the FFP will be officially supported by the FDA and other third party agencies that have approved it thus far [3]. There is applicable scientific evidence to certify the food as functional; however, there remain unknown factors that still require study. As indicated by Figure 4, the mediation of the FF's accreditation through the 16-step process is now approved by the FDA and recognized by an official governmental agency as such.

Step 14: The fourteenth step allows FF to be released to market after recommendation by third parties and approval by governmental agencies [3]. Depending on the stature of the FFP in the market upon release, some items may see an easier time as the release would almost act as an announcement. With items already heavily trafficked in the market, a release would act as categorization and documentation of values observed within the attributed product up until that point. By comparison, a food item that has not seen a market release yet, more likely processed FFs, would require a proper release. Thus, a proper and swift release is important so that products will not be overshadowed by already established items. At this point, the lowest grade associated with the product will be a ' $C$ ', as it has yet to be studied in uncontrolled environments on a large scale. Less than two decades ago, the FF market globally was estimated to be valued anywhere between 33 billion and 47.6 billion USD. Furthermore, coinciding with an estimated percentage share of the U.S. food market increase at the time, it is plausible that FFs have exceeded their 2-3\% market capacity. By the year 2008, the market share was estimated to double, supporting the assertion of an ever-expanding market [28]. Catering to the consumer and their desires is the best way to release a product to market. Due to much competition as alluded to previously, releasing FFPs to market needs to be a wellthought-out endeavor. When considering the taste and perception of FFs, some individuals are overly critical, not wishing to sacrifice flavors for a possible health benefit [29]. This way of thinking is to be expected if the producer of a product wishes to succeed. To overcome this, producers must be mindful of how their products are perceived versus others without health benefits, which can capitalize on taste. Interestingly, depending on the health claim and the magnitude of its capabilities, some people are willing to compromise taste for the possible benefit [30]. Regardless, a release to market is contingent on the way by which the food product is monitored afterward and adapted to the current climate.

Step 15: In the fifteenth step of the process, epidemiological studies will be conducted to better assess how a population resembling a market that is not proctored is affected by the implementation of the FFP. The dosage, efficacy, and safety will be analyzed further 
in this environment, a "real world" setting. Instead of having a specific population administered to, the FF will be evaluated in people of unconfirmed status involving specific afflictions [3]. By observing how the food product interacts in a population without strict limitations, a better analysis of its efficacy can be made as well as establish a line of trust with the public. Furthermore, any negative outcomes that could not previously be observed due to study design limitations may become apparent in this step. When considering the variety of epidemiological study designs, there are clear benefits as to which process is most applicable to the certification of FFPs. It was determined that a randomized controlled trial design is the most applicable to the updated 16 step process. The key benefit of such a design is the determination of a relationship amongst variables. In reference to chronic disease specifically, the randomized controlled trial design allows for preventative aspects of prevention and management characteristics to become known. Furthermore, the way in which precise research statistics are employed along with randomization help to secure the honesty and trustworthiness of the design in a data analysis framework. The cross-sectional study design accomplishes this but lack of temporality precludes a discussion of causality. Findings of crosssectional studies are largely correlational, in contrast to randomized controlled trial designs, which clearly assign causality [31]. By safeguarding randomization in the randomized controlled trial design, statistical integrity is maintained to a greater extent, allowing for trustworthy casual relationships to be identified. Once this stage is completed, the FFP may reapply for the approval of category ' $\mathrm{B}$ '.

Step 16: In the sixteenth step, to the discretion of the research party, aftermarket research is conducted on the perceived benefits of the food item [3]. An important aspect of the product's release to market is to maintain education and marketing, encouraging higher consumption rates. With this newer system, a stage such as this is best suited for post-approval of classification ' $B$ '. Despite variance in bioactive compound availability and efficacy, it is important to continually analyze how FF affects an open market. This is a more refined analysis when compared to the earlier epidemiological studies, as the dosage and use should be more optimal when compared to previous stages. Additionally, aftermarket research analyzes a much wider population, as there is little to no control besides legal regulations. It is important to analyze the product within the context of such a vast market, as it will help monitor the potential gap between the controlled studies and how the product actually affects an individual's health and relevant biomarker(s). Should discrepancies arise in efficacy or safety, the product will be re-evaluated. Furthermore, by finalizing the refinement of FFPs in this way, it may better establish means for analysis on another containing the same or similar bioactive compounds. When compared to previous publications, aftermarket research is relatively new. Prior, epidemiological studies were sanctioned as the final step [32]. In doing this, uncontrollable benefits or detriments cannot be observed before the food vehicle is released to markets as clinical studies take place in a monitored environment. Thus, a new step in aftermarket research needed to be added as a final precaution in the process in order to monitor the well-being of the public. As displayed throughout literature published by the FFC, the steps for certification are ever-evolving and becoming more focused as time persists. Upon completion, the product can now be subject to reapplication for the approval of being classified under category ' $A$ '. 
Categorization grades of functional food products: The Functional Food Center proposes a system in which hierarchy can be easily identified, approved FFPs may be ranked according to an alphanumeric system. This system will have multiple constituent factors which analyze the FFs being ranked and place them accordingly. Thus, a label of ' $A$ ' will denote a greater factor of support than a label of ' $B$ '. The system will range from ' $A$ ', the highest, to ' $C$ ', the lowest. Thus it will be synonymous with many common grading systems around the globe.

The first category, "Category C," is the basic classification for FFPs that have completed steps 1 through 14 for approval, because it remains to be studied in epidemiological and long-term aftermarket settings. Category C FFPs would be the minimum requirement for FF approval under the FFC's protocols and receive a logo of certification by the FFC as indicated in Figure 1.
The second category is defined as "Category B," and is a higher completed certification for FFPs that have completed steps 1 through 15 . This category includes epidemiological studies, but not aftermarket research. FFPs in this category will be a great standard for understanding the benefit to the general public and receive a logo of certification by the FFC as indicated in Figure 2.

The highest category, "Category A," is the premium rating for the approval of FFPs. Products that receive certification for Category A have gone through all mandatory steps 1 through 16, including epidemiological studies and aftermarket research. This is the gold standard for manufacturers for providing empirical evidence on the efficacy, legitimacy, and safety of the FFP. The logo of certification will clearly identify its excellence as the premium Category $A$ and its approval by the FFC as indicated in Figure 3.

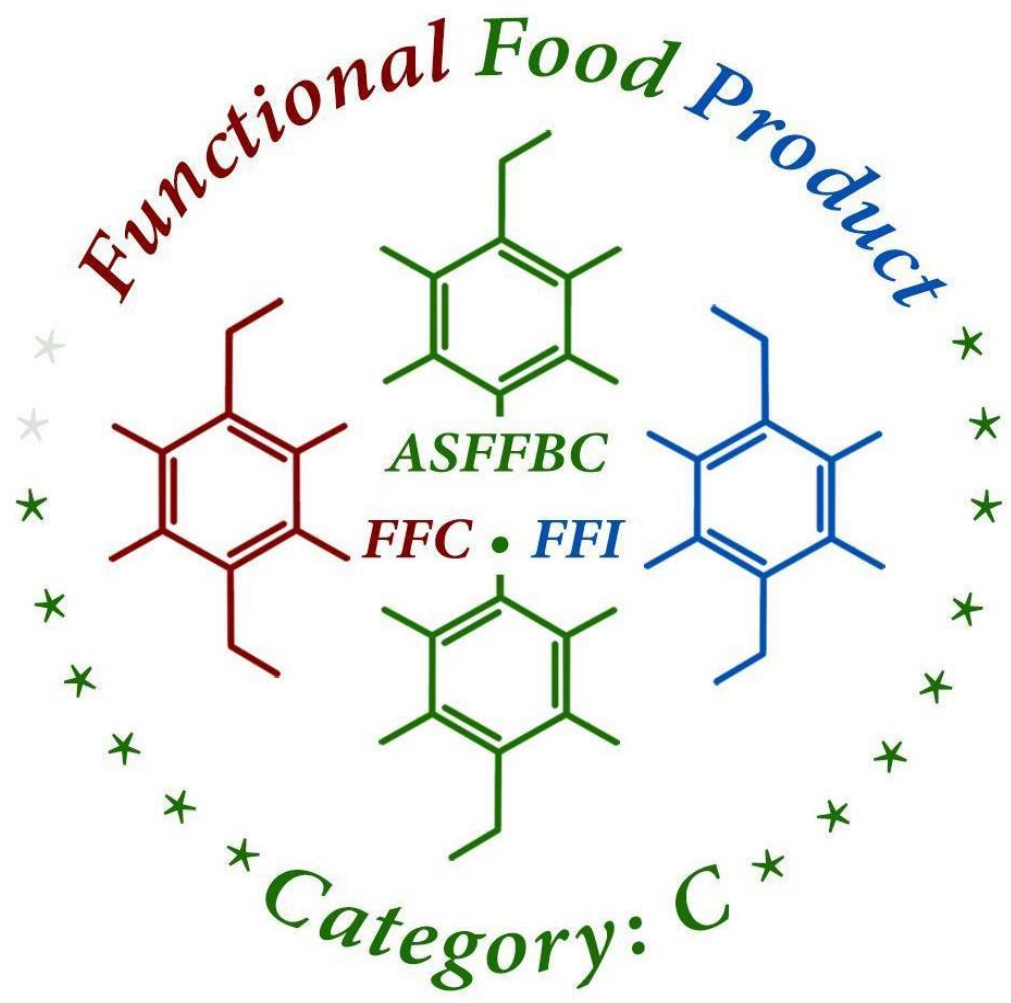

Figure 1. Logo for the certification of a Category $C$ functional food product. 


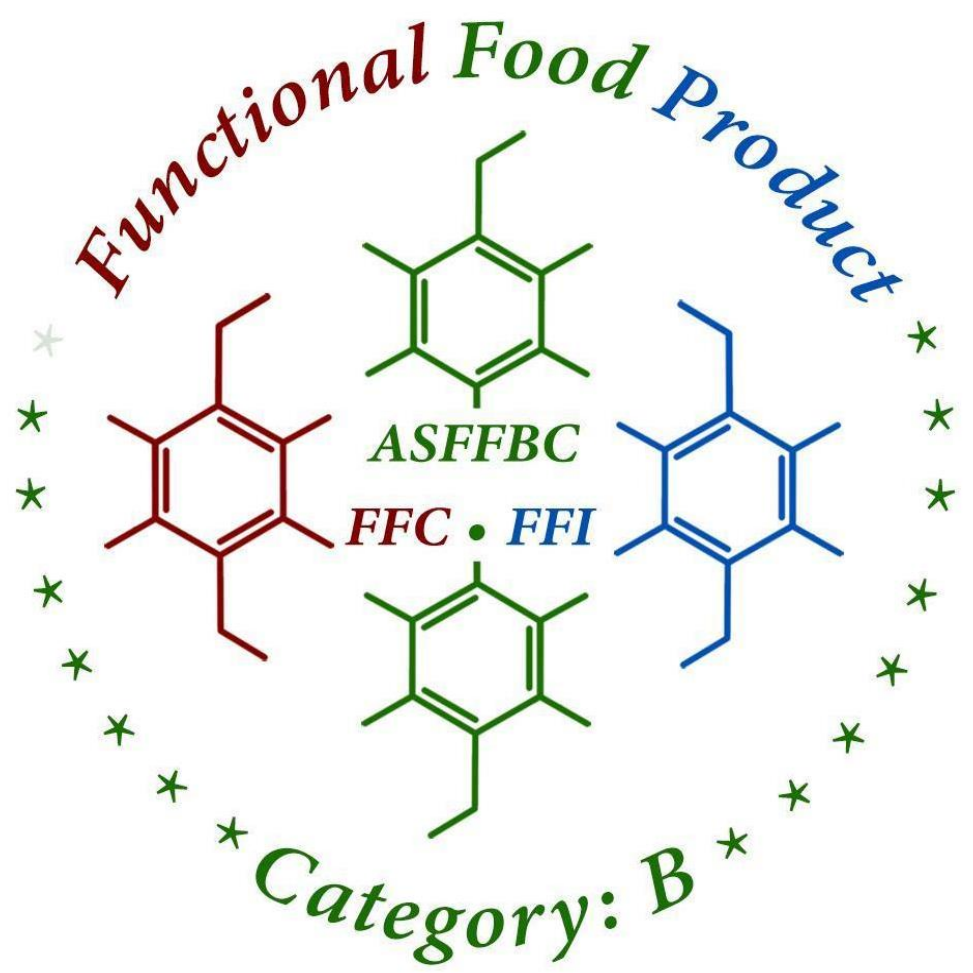

Figure 2. Logo for the certification of a Category B functional food product.

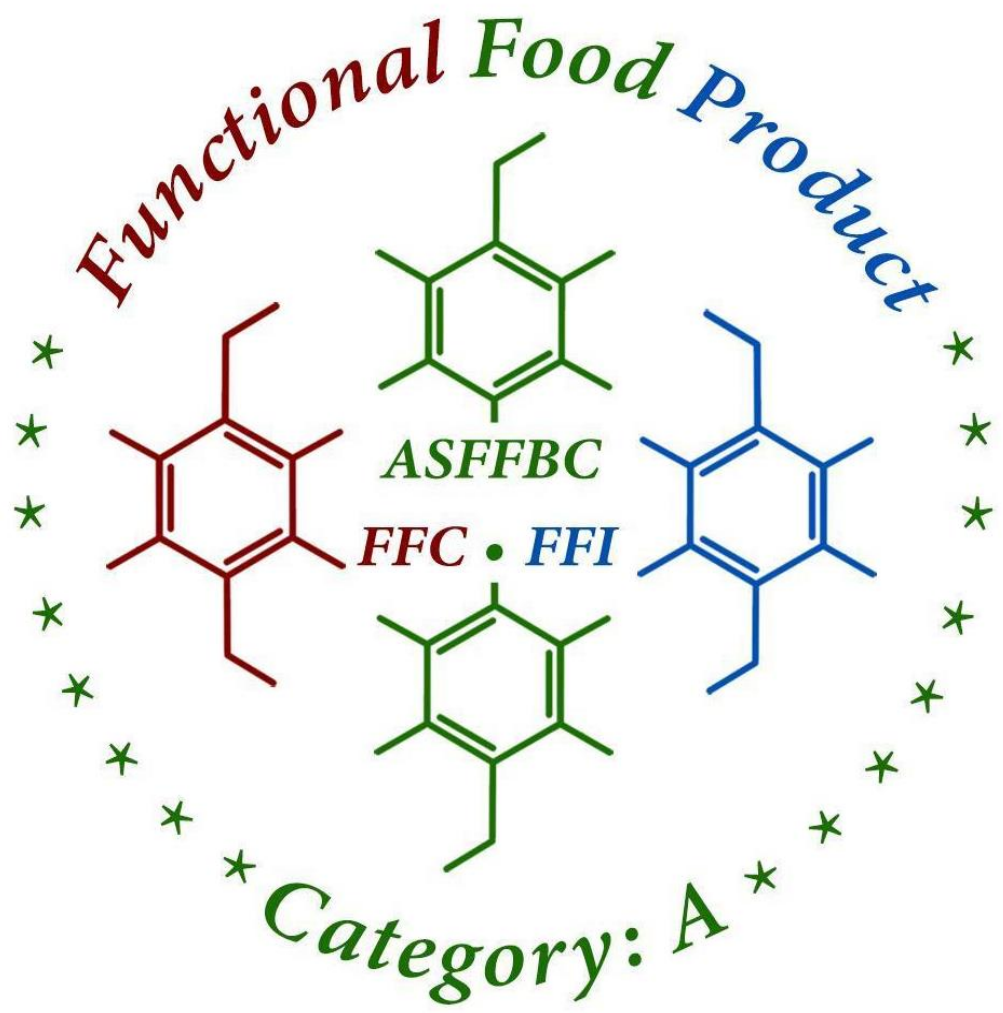

Figure 3. Logo for the certification of a Category A functional food product. 
Comparison of FOSHU, FDA, and FFC for the classification and regulation of functional foods: This section compares the evaluation and approval systems used by both the federal Food and Drug Administration (FDA) in the United States and the Food for Specified Health Uses (FOSHU) program in Japan, and describes the vision of the FFC in the classification and scientific regulation of FFs [33-34].

\section{Functional food regulation by FOSHU: When it comes} to the regulation of FFs, Japan is an excellent model. The Japanese government has established the "Food for Specified Health Uses" (FOSHU) as a regulatory system for FFs [5]. FOSHU refers to food with a claim that identifies a function benefiting health and is evaluated and approved by the government. FOSHU is part of the Foods with Health Claims regulations, which then enables health claim labeling on products that meet specific requirements [34]. FOSHU requires significant evidence from clinical studies. There are protocols and guidelines covering target subjects for enrollment, significant points, and parameters for symptoms. In both types of studies, healthy volunteers without medication are enrolled. Control of food uptake and life-style, plus recruitment of appropriate subjects under a good protocol, are important to establishing clear evidence of the active component. Approval requires 1) clear proof of effectiveness in the human body; 2) usage of nutritionally appropriate ingredients; 3) guarantee of compatibility with product specifications by the time of consumption; 4) and established quality control methods [34].

The FOSHU system requires the identification of the active ingredients as well as its mechanism of action. Therefore, the documentation which FOSHU requires is based on the identification and characterization of the active ingredient. Required documentations and the processes are described in detail by Yamada et al. (2008) [35]. The process for FOSHU certification begins by scientifically providing the efficacy of the active ingredient, including in vitro and in vivo tests, that describe the mode of action, together with human trial results that prove the efficacy of the product containing the active ingredient. FOSHU requires evidence for the safety of the FFP containing the active ingredient. Manufacturers must provide documents proving stability of the active ingredient, as well as for the FFP evaluated. FOSHU requires detail of the physical, chemical, and biochemical characteristics of the active ingredient, as well as the analytic methods used. The quantification of the active ingredient must be done with at least three random samples from different manufacturing dates or production lots [38]. The quality control methods implemented at the production facility of the product, including the ingredient and final product specifications and the equipment present at the production facility are submitted [35].

Although FOSHU is highly regarded as trustworthy, concerns still arise regarding its aftermarket framework. The regulation of FFs by FOSHU lacks aftermarket research, that is, research performed after the release of a FFP to investigate the effects it has on an uncontrolled environment that is commonly found to the general public [4]. Studies done post-market to assess FF efficacy can aid in the development of FFs as a science around the world.

2. Functional food regulation by FDA: The classification and regulation of FFs in the United States greatly differs from the FOSHU certification system in Japan. In the United States, the Food and Drug Administration (FDA) is a governing body responsible for enforcing food and drug safety protocols under the Federal Food, Drug, and Cosmetic Act (FFDCA) and later, through the 1994 Dietary Supplement Health and Education Act (DSHEA) [36]. Unfortunately for the development of FFs, there is no formal regulatory category for FFs as the FFDCA does not provide a statutory definition of FFs. Food categories for which federal definitions exist include food and dietary supplement, but not FF. With an absence of a FF 
category, the regulation of potential FFs is currently accomplished through existing regulations.

Foods and supplements are regulated by the FDA by their intended use, which is determined by health claims and accompanying label information [36]. FDA categorizes the intended use for the labeling of food and health claims into four types: nutrient content claims, authorized health claims, qualified health claims, and structure/function health claims. Nutrient content claims describe the level of a nutrient in the product, using terms such as free, high, and low, or they compare the level of a nutrient in a food to that of another food, using terms such as more, reduced, and lite [36]. Authorized health claims provide the use in food labeling of health claims that characterize a relationship between a food, a food component, or dietary ingredient and risk of a disease. For example, "adequate calcium throughout life may reduce the risk of osteoporosis" [36]. Qualified health claims may be used when there is emerging evidence for a relationship between a food component and reduced risk of a disease, but the evidence is not well enough established to meet the scientific agreement standard. This is problematic because a qualified health claim may not provide empirical evidence, but nevertheless allows the product to remain marketable for health purposes [39]. Structure/function health claims may describe the role of a nutrient or dietary ingredient intended to affect the normal structure of the human body, for example, "calcium builds strong bones." In addition, they may characterize the means by which a nutrient or dietary ingredient acts to maintain such structure or function, for example, "fiber maintains bowel regularity," or "antioxidants maintain cell integrity" [36]. In order to verify food claims, the FDA focuses on research involving the administration of food compounds to animals and humans. Claims must then be approved under "Significant Scientific Agreement" after which FDA officials rank claims based on the strength of the scientific evidence behind them [37].

\section{Functional food regulation by FFC: The concept of FFs} requires systematic and methodical regulation, notably in the United States, where there is no acknowledged or official classification for FFs [3]. The FFC has proposed that the regulation of FFs as most adequately accomplished by using a multi-step procedure that focuses on bioactive compounds as the backbone for approval [12]. These bioactive compounds are primary and secondary metabolites of nutritive and non-nutritive natural components that generate health benefits by preventing or managing chronic disease or its symptoms. The study on the protective and health promoting effects of bioactive compounds have made these components particularly important for the categorization of FFs [10].

Table 2. Comparison of the established functional food procedure by FOSHU and the proposed methodology by the FFC

\begin{tabular}{|c|c|c|}
\hline Parameters & FOSHU & FFC \\
\hline Contains causal compounds & $\begin{array}{l}\text { Active ingredients with established } \\
\text { mechanism, characteristics, and stability }\end{array}$ & Similar in concept to bioactive compounds \\
\hline $\begin{array}{l}\text { Required establishment of } \\
\text { bioactive mechanism }\end{array}$ & Yes & Yes \\
\hline $\begin{array}{l}\text { Establishment of dosage and } \\
\text { non-toxic quantities of the } \\
\text { bioactive compound }\end{array}$ & Yes & Yes \\
\hline $\begin{array}{l}\text { Possibility of FF with or without } \\
\text { proven bioactive compounds }\end{array}$ & $\begin{array}{l}\text { The active ingredient(s) must be identified } \\
\text { and be measurable }\end{array}$ & $\begin{array}{l}\text { It is absolutely necessary to find bioactive } \\
\text { compound(s) and ratio of compounds if there } \\
\text { are more than one bioactive compound } \\
\text { responsible for a health benefit }\end{array}$ \\
\hline
\end{tabular}




\begin{tabular}{|c|c|c|}
\hline Parameters & FOSHU & FFC \\
\hline $\begin{array}{l}\text { Contains definition of bioactive } \\
\text { compounds }\end{array}$ & $\begin{array}{l}\text { The word equivalent to "bioactive } \\
\text { compound" is not officially defined, but } \\
\text { contextually it is obvious that the Japanese } \\
\text { word "active ingredients" refers to the } \\
\text { causal compound that provides health } \\
\text { benefits }\end{array}$ & Yes, and FFC suggested [40] \\
\hline $\begin{array}{l}\text { Necessary definition of } \\
\text { functional foods for } \\
\text { governmental agencies, } \\
\text { manufacturers, and consumers }\end{array}$ & $\begin{array}{l}\text { The equivalent of "Functional Foods" is } \\
\text { "Foods with Health Claims", which includes } \\
\text { FOSHU }\end{array}$ & Yes, and FFC suggested [40] \\
\hline $\begin{array}{l}\text { Conditions and disease } \\
\text { identified by FFP to reduce the } \\
\text { risk or mitigate disease }\end{array}$ & $\begin{array}{l}\text { 1. Special health condition } \\
\text { 2. Maintenance of health } \\
\text { 3. Cannot mention disease names (with } \\
\text { exception of risk reduction: FOSHU is not } \\
\text { medicine) }\end{array}$ & $\begin{array}{l}\text { 1. General health } \\
\text { 2. Chronic diseases } \\
\text { 3. Reduce the risk of viral diseases }\end{array}$ \\
\hline $\begin{array}{l}\text { Evaluation of new delivery } \\
\text { vehicle with each new FFP }\end{array}$ & $\begin{array}{l}\text { Yes, the effectiveness is tested on the final } \\
\text { product basis, and each product will be } \\
\text { evaluated by CAA under the FOSHU } \\
\text { regulation. }\end{array}$ & $\begin{array}{l}\text { Yes, necessary evaluate all steps since new } \\
\text { product is a new environment and bioactive } \\
\text { compounds mechanism and activity might be } \\
\text { changed }\end{array}$ \\
\hline Ideal intervention & Randomized controlled trials & Randomized controlled trials \\
\hline Measurement of the outcome & $\begin{array}{l}\text { Use an indicator that is appropriate for the } \\
\text { purpose and widely considered clinically and } \\
\text { nutritionally meaningful }\end{array}$ & $\begin{array}{l}\text { Find the biomarker that can be tested via that } \\
\text { mechanism or pathway, which indicates } \\
\text { effectiveness of the bioactive compound }\end{array}$ \\
\hline $\begin{array}{l}\text { In vitro, in vivo, and clinical } \\
\text { studies necessary for approval } \\
\text { as FF product }\end{array}$ & Yes & Yes \\
\hline $\begin{array}{l}\text { Requirement of specialized } \\
\text { label }\end{array}$ & Yes & Yes \\
\hline After market studies & No & Yes \\
\hline $\begin{array}{l}\text { Publishing investigation on FFP } \\
\text { in a journal }\end{array}$ & No & Yes \\
\hline $\begin{array}{l}\text { Report investigation results in } \\
\text { international conferences } \\
\text { related to the field FFC }\end{array}$ & No & Yes \\
\hline Government approval of FFP & $\begin{array}{l}\text { Yes. However, Foods with Function Claim are } \\
\text { not evaluated or approved by CAA, and } \\
\text { therefore they are not FOSHU. }\end{array}$ & Yes \\
\hline Beneficiaries of the FF product & $\begin{array}{l}\text { 1. Manufacturers } \\
\text { 2. Government } \\
\text { 3. Consumers }\end{array}$ & $\begin{array}{l}\text { 1. Scientist(s) who come up with formulation } \\
\text { 2. manufacturers } \\
\text { 3. government } \\
\text { 4. consumer }\end{array}$ \\
\hline
\end{tabular}


The FFC's proposed regulation of FFs resolves the two issues that arise within the FDA and FOSHU processes. First, a formal category of FFs will be defined, and second, epidemiological studies and after-market research will be a required step for premium classification. While the FDA does recognize the efficacy of certain food compounds having disease-related health benefits, they have not recognized FFs as a separate category [1]. The FFC multistep procedure links the consumption of FFs with health claims that are based on published, empirical evidence. Regarding FOSHU, the problem of a lack of epidemiological studies and aftermarket research is resolved under our proposed system. The aftermarket research for a FFP will be a required step for manufacturers seeking the premium level of certification. An outline for how approval/ recommendation would be completed is shown in the above Figure 4 . The proposal is an open system, meaning it may be modified in the future. As the FFC starts to implement its recommendations to the FDA, then discussion will further need to follow dictating the evolution of the proposal.

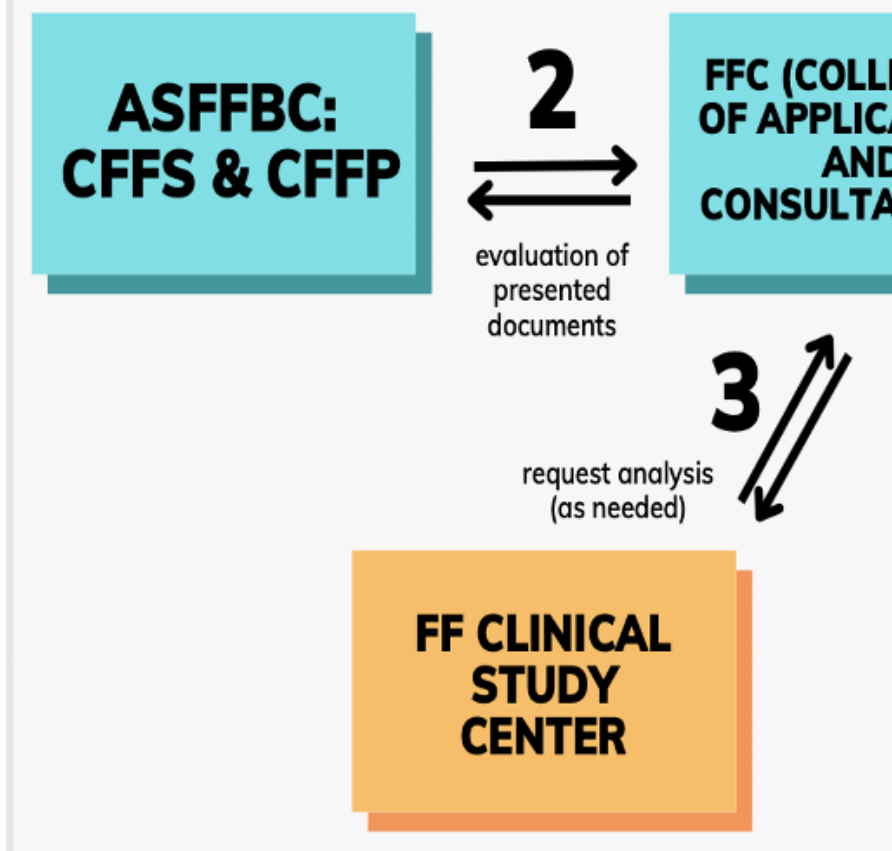

Figure 4. General overview of the mediation process

An outline for how approval/recommendation would be completed is shown in the above Figure 4. The proposal is an open system, meaning it may be modified in the future. As the FFC starts to implement its recommendations to the FDA, then discussion will further need to follow dictating the evolution of the proposal.

An application consisting of the initial steps and appropriate detailed documentation would be sent to the FFC, which in turn would be communicated to various certified functional food scientists and certified functional food professionals. Collaborative members from ASFFBC may also be used for each specific case for the potential certified functional foods (CFFs) and certified functional food products (CFFP). After the verification and approval of required documents, as well as, functional food science publications, the FFC will be able to provide recommendations about the continuation of the case. This preliminary screening will improve the efficiency for the applicants to create a FFP, 
prior to sending data to the FDA. Additionally, this preliminary screening of applications and appropriate consultations with applications will save governmental agencies' time to make decisions upon the classification of potential FFP and the approval or disapproval of the product.

After a preliminary screening, the FDA may evaluate forms and research done by the applicant, confirm or deny the validity of the FFP and allow it to be marketed with an appropriate label by the applicant. The FFP can now be graded as ' $C$ ' and established as a FF on the market. For higher grades of classification in the future, the continuation of evidence including epidemiological studies and after-market research will need to be completed upon applying for grades of ' $\mathrm{B}$ '

and ' $A$ '. The FFC's role in the approval process is to assist both the applicant and the appropriate governmental agency be as efficient as possible. The collaboration within the network of functional food professionals can be helpful for applicants as well as for governmental agencies in the certification process, but final approval should be the responsibility of a governmental agency. It is important to note that applicants may also apply to the FDA directly.

As a pioneer in the field of functional food science, it is justifiable that the official approval process is in collaboration with the FFC. The FFC connects a global network of professionals in the field of functional food science as demonstrated by hosting twenty-nine international conferences in the field of functional food science, and is scheduled for two upcoming international conferences for 2022 and 2023 [39]. Additionally, the FFC has published nine textbooks, published several peerviewed journals, and developed online classes to teach students. The FFC has created several forms of certification process for those who would like to become certified functional food scientists and professionals. The FFC is the most knowledgeable organization for collaborating with government agencies because the FFC has developed the methodology and produced the classification, and categorization, for functional foods. The FFC's initial screening of scientific work and documents from applicants will make the case for approval much stronger.

This approval process will benefit our society by having healthy and functional food options in combination with our standard medicine practices. Government agencies will save money in health care costs, scientists will get reward for their sound scientific work in this modern and interdisciplinary field, the food industry will be rewarded for producing healthy and functional foods, and most importantly this will benefit millions of lives making longer lifespan and happier of people who will consume specific functional food product for reducing risk of diseases as well as for wellbeing

\section{Open-ended expansion}

This system is left relatively open and flexible. Should the need for new categories be present in the future, then adaptations can be made as needed. This is to allow for a system that can grow as the field of FF does. As demonstrated in Figure 5 below, the newest steps of our proposal have expanded upon previous ideas of the regulatory process. In the future, slight alterations to the current process may be adjusted; however, the general outline is foundational. Additional steps are not always necessary. As mentioned previously, the order in which FFs are established will remain relatively consistent as there are prerequisites for each step; however, some instances may be allowed to shift slightly. 


\section{Functional Food Science: Revised Proposed Process Outline}
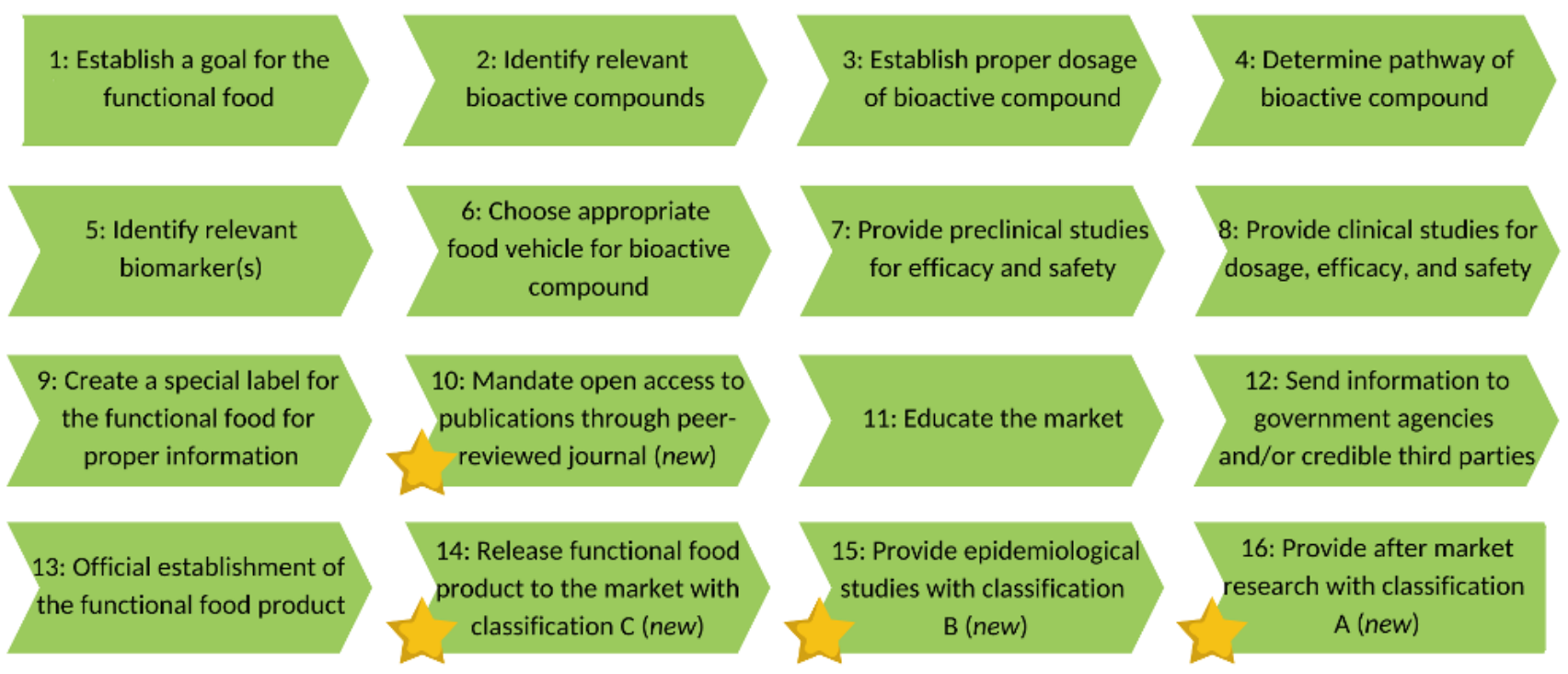

Figure 5. The highlight of our newest steps to develop functional food products and bring them to the market

\section{CONCLUSION}

In providing a new system by which functional food products can be classified, A, B, or C, the Functional Food Center can better communicate the prospects of symptom management and risk reduction on the part of food items to the public and official agencies. Notably, the system is comparative in rigor to the FOSHU system utilized in Japan, allowing for justification of functional food expansion in the U.S. An easy and digestible classification system such as the one proposed offers food producers another means through which to market their product, and consumers' knowledge can be leveraged to better understand how certain food products could make a substantial and beneficial impact on their lives. Furthermore, building on already established claims made by the FDA concerning specific food items affords the Functional Food Center and items in question a justification for expansion of knowledge concerning their bioactive compounds. The new system emphasizes greater transparency, as it implements a suggestion for open access scrutinization. This new step will expand knowledge and literature, as data for a specific FFPs will be subject to peer evaluation. In being transparent with official agencies and the average consumer, safety can be better ensured, providing sound justification for field expansion. The uniqueness of this newer process lies in this aspect. Jointly with better means of communication, FF can now be held to an even higher standard. Furthermore, when compared to previous plans proposed by the FFC, the evolution of thought is evident and clear. This proposal sees slight adjustments to the already instated functional food certification system. While not a total overhaul, the addition of another step in the form of open-access dictates a subsequent proposal in order to affirm its status as a standard

Abbreviations: ASFFBC: Academic Society of Functional Foods and Bioactive Compounds; CAA: Consumer Affairs Agency; CDC: Center for Disease Control; DSHEA: Dietary Supplement Health and Education Act; FFC: Functional Food Center; FFP: Functional Food Product; FOSHU: Foods for Specific Health Uses; FDA: Food and Drug Administration; FSIS: Food and Safety Inspection Services; FFDCA: Federal Food, Drug, and Cosmetic Act; FFI: Functional Food Institute; FF: Functional Food; CFF: 
Certified Functional Food; CFFP: Certified Functional Food Product

Acknowledgement: We would like to thank Tim Roller, Michelle Lee and Silvia Sanchez for helping us create infographics, images and tables.

Conflicts of Interest: There are no conflicts of interest associated with this study.

\section{Authors' contribution: The original idea (Classification} and Regulation of Functional Foods proposed by Functional Food Center) was conceived by DM and was discussed with ME and TL. ME and TL collected data and wrote the manuscript. DM advised, participated in writing and editing manuscript. All authors read and approved the final version of the manuscript

\section{REFERENCES:}

1. Raghupathi W, Raghupathi V. An Empirical Study of Chronic Diseases in the United States: A Visual Analytics Approach. Int J Environ Res Public Health. 2018;15(3):431. Published 2018 Mar 1.. https://doi.org/10.3390/ijerph15030431

2. Sadohara R., Martirosyan D. Functional Food Center's vision on functional foods definition and science in comparison to FDA's health claim authorization and Japan's Foods for Specified Health Uses. Functional Foods in Health and Disease 2020. 10(11): 465-481. doi: https://www.doi.org/10.31989/ffhd.v10i11.753

3. Martirosyan D., Kanya H., Nadalet C. Can functional foods reduce the risk of disease? Advancement of functional food definition and steps to create functional food products. Functional Foods in Health and Disease 2021; 11(5): 213-221. doi https://www.doi.org/10.31989/ffhd.v11i5.788

4. Adany, A. Kanya, H. Martirosyan, D. Japan's health food industry: An analysis of the efficacy of the FOSHU system. Functional Foods in Health and Disease 2021. 4(4):63-78. doi: https://www.doi.org/10.31989/bchd.v4i4.795

5. Shimizu T. Health claims on functional foods: the Japanese regulations and an international comparison. Nutr Res Rev. 2003;16(2):241-252. doi:10.1079/NRR200363

6. Ministry of Health, Labour, and Welfare [https://www.mhlw.go.jp/english/topics/foodsafety/fhc/02.ht ml] retrieved January 5, 2022

7. Ellwood KC, Trumbo PR, Kavanaugh CJ. How the US Food and
Drug Administration evaluates the scientific evidence for health claims. Nutr Rev. 2010;68(2):114-121. https://doi.org/10.1111/j.1753-4887.2009.00267.x

8. Martirosyan D., von Brugger J., Bialow S. Functional food science: Differences and similarities with food science. Functional Foods in Health and Disease 2021. 11(9): Page Number: 408-430. doi: https://www.doi.org/10.31989/ffhd.v11i9.831

9. Singharaj B, Pisarski K, Martirosyan DM. Managing hypertension: relevant biomarkers and combating bioactive compounds. 2020; 7(6):442-461. doi: https://doi.org/10.31989/ffhd.v7i6.324

10. Martirosyan D.M., Singh J. A new definition of functional food by FFC; what makes a new definition unique?. 2015; 5(6):209223. doi: 10.31989/ffhd.v5i6.183

11. Konstantinidi $M$, Koutelidakis AE. Functional Foods and Bioactive Compounds: A Review of Its Possible Role on Weight Management and Obesity's Metabolic Consequences. Medicines (Basel). 2019;6(3):94. Published 2019 Sep 9. https://doi.org/10.3390/medicines6030094

12. Ntrigiou V., Ntrigios I., Rigopoulos N., Dimou C., Koutelidakis A. Functional food consumption correlates with anthropometric characteristics and body composition in healthy adults. Curr. Top. Nutraceut. Res. 2019;18:279-288.

13. Elmaliklis I.N., Liveri A., Ntelis B., Paraskeva K., Goulis I., Koutelidakis A. Increased Functional Foods' Consumption and Mediterranean Diet Adherence May Have a Protective Effect in the Appearance of Gastrointestinal Diseases: A Case-Control Study. Medicines. 2019;6:50 https://doi.org/10.3390/medicines6020050

14. Koutelidakis A., Dimou C. The effects of functional food and bioactive compounds on biomarkers of cardiovascular diseases. In: Martirosyan D., editor. Functional Foods Text Book. 1st ed. Functional Food Center; Dallas, TX, USA: 2016. [(accessed on 15 February 2019)]. pp. 89-117.

15. Banwo, K., Olojede, A. O., Adesulu-Dahunsi, A. T., Verma, D. K., Thakur, M., Tripathy, S., Singh, S., Patel, A. R., Gupta, A. K., Aguilar, C. N., and Utama, G. L. (2021, August 23). Functional importance of bioactive compounds of foods with potential health benefits: A review on recent trends. Food Bioscience. doi: 10.1016/j.fbio.2021.101320

16. Marcela Bromberger Soquetta, Lisiane de Marsillac Terra and Caroline Peixoto Bastos (2018) Green technologies for the extraction of bioactive compounds in fruits and vegetables, CyTA - Journal of Food, 16:1, 400-412, https://doi.org/10.1080/19476337.2017.1411978

17. Sasidharan S, Chen Y, Saravanan D, Sundram KM, Yoga Latha L. Extraction, isolation and characterization of bioactive 
compounds from plants' extracts. Afr J Tradit Complement Altern Med. 2011;8(1):1-10.

18. Biesalski HK, Dragsted LO, Elmadfa I, et al. Bioactive compounds: definition and assessment of activity. Nutrition. 2009;25(11-12):1202-1205.

https://doi.org/10.1016/j.nut.2009.04.023

19. Ronis MJJ, Pedersen KB, Watt J. Adverse Effects of Nutraceuticals and Dietary Supplements. Annu Rev Pharmacol Toxicol. 2018;58:583-601. doi:10.1146/annurev-pharmtox010617-052844

20. Serrano JC, Jove M, Gonzalo H, Pamplona R, Portero-Otin M. Nutridynamics: mechanism(s) of action of bioactive compounds and their effects. Int J Food Sci Nutr. 2015;66 Suppl 1:S22-S30. https://doi.org/10.3109/09637486.2015.1035231

21. Strimbu K, Tavel JA. What are biomarkers?. Curr Opin HIV AIDS. 2010;5(6):463-466, https://doi.org/10.1097/COH.0b013e32833ed177

22. Rafter, J. (2002). Scientific basis of biomarkers and benefits of functional foods for reduction of disease risk: Cancer. British Journal of Nutrition, 88(S2), S219-S224. https://doi.org/10.1079/BJN2002686

23. Otunola GA, Martirosyan D. Choosing Suitable Food Vehicles for Functional Food Products. 2021; 11(2): 44-55. doi: https://www.doi.org/10.31989/ffhd.v11i2.764

24. Gur J., Mawuntu M., Martirosyan D. FFC's Advancement of Functional Food Definition. Functional Foods in Health and Disease 2018; 8(7): 385-397 doi: https://doi.org/10.31989/ffhd.v8i7.531

25. T. Muller L, Ruffieux $B$. What makes a front-of-pack nutritional labelling system effective: The impact of key design components on food purchases. Nutrients. 2020;12(9):2870. https://doi.org/10.3390/nu12092870

26. Wu W, Zhang A, van Klinken RD, Schrobback P, Muller JM. Consumer Trust in food and the Food System: A critical review. Foods. 2021;10(10):2490. doi:10.3390/foods 10102490

27. Papadopoulos A, Sargeant JM, Majowicz SE, et al. Enhancing public trust in the Food Safety Regulatory System. Health Policy. 2012;107(1):98-103. https://doi.org/10.1016/j.healthpol.2012.05.010

28. Siró I, Kápolna E, Kápolna B, Lugasi A. Functional Food. Product development, marketing and Consumer Acceptance-a review. Appetite. 2008;51(3):456-467 https://doi.org/10.1016/i.appet.2008.05.060

29. Verbeke W. Functional Foods: Consumer willingness to compromise on taste for Health? Food Quality and Preference.
2006;17(1-2):126-131.

https://doi.org/10.1016/j.foodqual.2005.03.003

30. Urala N, Lähteenmäki L. Attitudes behind consumers' willingness to use functional foods. Food Quality and Preference. 2004;15(7-8):793-803.

https://doi.org/10.1016/j.foodqual.2004.02.008

31. Agarwal P., Rutter E., Martirosyan D.M. Analysis of contemporary epidemiological study research design formats on addressing functional food efficacy. Functional Food Science 2021; 1(12): 97-116. doi: https://www.doi.org/10.31989/ffs.v1i12.882

32. Liufu J., Martirosyan D. FFC's Advancement and the Establishment of Functional Food Science. 2020; 10(8):344356.https://doi.org/10.31989/ffhd.v10i8.729

33. Ross S. Functional foods: the Food and Drug Administration perspective. Am J Clin Nutr. 2000;71(6 Suppl):1735S-42S. https://doi.org/10.1093/ajcn/71.6.1735S

34. Kazuhiko Yamada, Natsuko Sato-Mito, Junichi Nagata, Keizo Umegaki, Health Claim Evidence Requirements in Japan, The Journal of Nutrition, Volume 138, Issue 6, June 2008, Pages 1192S-1198S, https://doi.org/10.1093/jn/138.6.1192S

35. Shun Iwatani, Naoyuki Yamamoto,Functional food products in Japan: A review, Food Science and Human Wellness, Volume 8 , Issue 2, 2019, Pages 96-101, https://doi.org/10.1016/j.fshw.2019.03.011.

36. Label Claims for Conventional Foods and Dietary Supplements [https://www.fda.gov/food/food-labeling-nutrition/labelclaims-conventional-foods-and-dietary-supplements] Retrieved December 30, 2021.

37. Guidance for Industry: Evidence-Based Review System for the Scientific Evaluation of Health Claims. January 2009. [https://www.fda.gov/regulatory-information/search-fdaguidance-documents/guidance-industry-evidence-basedreview-system-scientific-evaluation-health-claims]. Retrieved January 5, 2022.

38. Martirosyan D., Miller E., Bioactive Compounds: The Key to Functional Foods. Bioactive Compounds in Health and Disease 2018; 8(7):36-39. https://doi.org/10.31989/bchd.v1i3.539

39. Functional Food Center Inc. January 2022. [https://www.functionalfoodscenter.net/certification.html]. Retrieved January 7, 2022.

40. Ortiz M, Martirosyan D: Classification of "healthy" food by quantification of nutrient content based on functional and therapeutic effect on human health. In: Martirosyan D (ed) Basic Principles of Functional Food Science (Volume 6), 1st edn. Food Science Publisher, Dallas, Texas, 2018: 238-256 